\title{
Criticality and Transmission of Information in a Swarm of Cooperative Units
}

\author{
Fabio Vanni, ${ }^{1}$ Mirko Luković, ${ }^{2}$ and Paolo Grigolini ${ }^{1}$ \\ ${ }^{1}$ Center for Nonlinear Science, University of North Texas, P.O. Box 311427, Denton, Texas, 76203, USA \\ ${ }^{2}$ Max Planck Institute for Dynamics and Self-Organization, 37073 Göttingen, Germany
}

(Received 19 May 2011; published 12 August 2011)

\begin{abstract}
We show that the intelligence of a swarm of cooperative units (birds) emerges at criticality, as an effect of the joint action of frequent organizational collapses and of spatial correlation as extended as the flock size. The organizational collapses make the birds become independent of one another, thereby allowing the flock to follow the direction of the lookout birds. Long-range correlation violates the principle of locality, making the lookout birds transmit information on either danger or resources with a time delay determined by the time distance between two consecutive collapses.
\end{abstract}

DOI: 10.1103/PhysRevLett.107.078103

In the recent few years, there has been intense activity to explain why a swarm of birds behaves as a single individual $[1,2]$. How is it possible that when a predator comes, the swarm changes direction to escape from danger? How is it possible that a subset of a swarm becoming aware of the right direction toward a resource [3] convinces the whole flock to pursue that specific course? In which sense, using a metaphor made popular by Couzin [4], can we interpret the swarm as a cognitive mind?

The main purpose of this Letter is to prove that this form of intelligence is the effect of the joint action of frequent organizational collapses, allowing the single birds to recover independence of the others, and of a correlation length as extended as the flock size. Although the environment perceiving units are a small fraction of the total number of units, they exert a determinant action on the swarm during the short rearrangement phase after an organizational collapse, which makes each unit free to select a new direction. This freewill condition allows the swarm to select the new flying directions that are transmitted to all the units by the few danger- or resource-perceiving birds, thanks to the criticality-induced long-range correlation. The connection between dynamic instability and information transfer has been recently observed in locust nymphs [3]. The results of this Letter confirm the importance of this observation, establishing at the same time that the information transfer is made possible by the nonlocal nature of the criticality condition, with a time delay depending on the time distance $\tau$ between two consecutive organizational collapses. The correlation length between birds becomes as extended as the finite swarm size, thereby allowing the lookout birds to transmit their flying direction to the whole swarm. Thus, the mean value $\langle\tau\rangle$, proportional to the correlation length, remains finite.

To afford a convincing proof that the swarm's intelligence is determined by the joint action of organizational collapses and criticality-induced nonlocality, we proceed in two main steps. In the first step we use a model of bird organization, referred to in this Letter as the bird model, to
PACS numbers: 87.23.Cc, 64.60.fd, 89.70.-a, 89.75.Hc

illustrate the concept of temporal complexity. The second step is based on a simpler model, where the relative positions of the birds are fixed and they have only to choose between either flying to the right or the left.

We use the occurrence of organizational collapses to define temporal complexity [5] as follows. Let us set the origin of time at the moment of a failure occurrence, and let us consider the probability that another failure occurs in the small time interval $[\tau, \tau+d \tau]$ :

$$
d p=\psi(\tau) d \tau .
$$

Temporal complexity [5] is established by examining the asymptotic time limit of $\psi(\tau)$, which is expected [6] to be the same as that of

$$
\psi(\tau)=(\mu-1) \frac{T^{\mu-1}}{(\tau+T)^{\mu}} .
$$

Note that the parameter $T$ is the minimal recovery time and that Eq. (2) is a generic expression for a system in a critical state. The mean time distance between two consecutive collapses is given by

$$
\langle\tau\rangle=\frac{T}{(\mu-2)},
$$

for $\mu>2$. The ideal condition of temporal complexity corresponds to $\mu<2$, making $\langle\tau\rangle$ diverge, thereby generating the nonergodic condition [7]. There are reasons to believe that this condition may be a general property of physiological systems, playing an important role for the transfer of information from one to another complex system $[8,9]$.

To prove that a flock of birds at criticality generates temporal complexity, let us adopt the algorithm proposed by Vicsek and co-workers [10]. This model gives the prescription for each single bird to take a direction equal to the average of its nearest neighbors' orientations, inside a given circle of interaction of radius $r$, with some uncertainty represented by a white noise of intensity $\eta$. The signature of cooperative behavior is given by the intensity of the global speed, defined by 


$$
\phi_{a}(t)=\frac{1}{N v_{0}}\left|\sum_{k=1}^{N} v_{0} e^{i \theta_{k}(t)}\right|
$$

$\theta_{k}(t)$ is the angle specifying the flight direction, $N$ is the number of birds, and $\eta$ is the parameter that has to be tuned to make the system critical.

To detect the occurrence of organizational collapses, we monitor the alignment of the swarm looking for the ideal condition of a vanishing $\phi_{a}(t)$. Actually, we find that, although $\phi_{a}(t)=0$ never occurs, from time to time $\phi_{a}(t)$ becomes smaller than a threshold that we arbitrarily establish to signal the transition from the organized to the nonorganized state. We make the assumption that crossing this threshold marks the occurrence of an organizational collapse establishing the swarm's free will. In Fig. 1, we plot the survival probability $\Psi(t) \equiv \int_{t}^{\infty} d t^{\prime} \psi\left(t^{\prime}\right)$, where $\psi(\tau)$ is the distribution density of the time distances between two consecutive collapses. We see that at criticality $\psi(\tau)$ is proportional to $1 / \tau^{\mu}$, with $\mu$ close to 1.35 .

The one-dimensional version of this model shows a behavior qualitatively similar to that of the locusts of Ref. [3], characterized by abrupt jumps from one to the opposite flying direction. In this case a direction change marks with no ambiguity the occurrence of an organizational collapse and proves that the reorganization time is negligible compared to $\langle\tau\rangle$. Furthermore, recovering $\mu=$ 1.35 [see $\Psi(t)$ of Fig. 3] suggests universality.

We note that, although at criticality the waiting time distribution density tends to fit the theoretical definition of temporal complexity of Eq. (2), at $t \sim 10000$ in the case of Fig. 1, the inverse power law is interrupted by a fast exponential drop. This is a consequence of the swarm's size being finite. We find numerically that the exponential truncation occurs at larger and larger times with increasing the size of the swarm. In the specific case of Fig. 1, we find that $\langle\tau\rangle \approx 10^{4}$. The effective mean velocity of the swarm is $V \approx 0.004$. The swarm is contained in a square of size

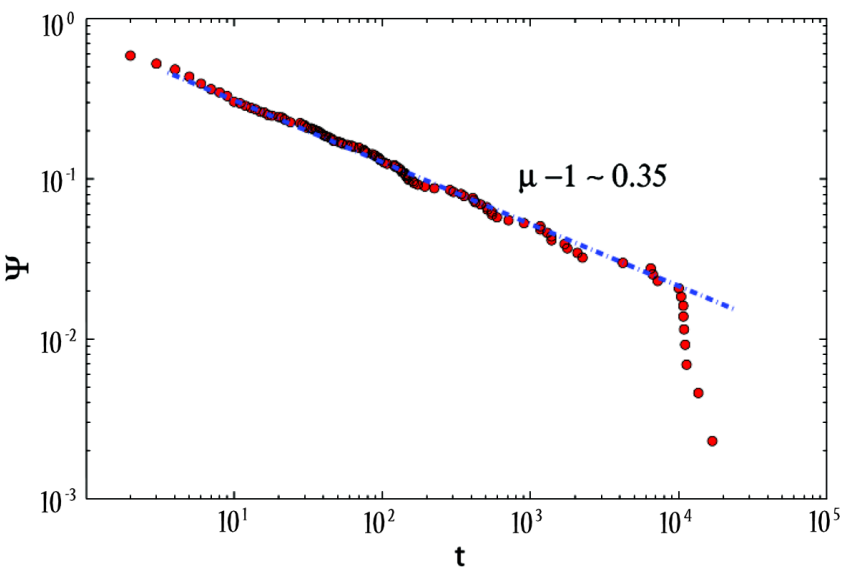

FIG. 1 (color online). Survival probability $\Psi(t)$ as a function of time. The occurrence of collapses is detected by using the threshold of Fig. 2.
$L=40$. Thus, it takes the time $T \approx 1 \times 10^{4}$ for the swarm to travel a distance of the order of its size. We see that during this time the swarm undergoes at least one collapse. The numerical calculations yield

$$
\frac{\left\langle\tau_{1}\right\rangle}{\left\langle\tau_{2}\right\rangle} \approx \frac{L_{1}}{L_{2}}
$$

where $L_{1}$ and $L_{2}$ indicate two different swarm sizes and $\left\langle\tau_{1}\right\rangle$ and $\left\langle\tau_{2}\right\rangle$ the corresponding mean time distances between two consecutive organizational collapses. Note that this is so because at criticality both correlation length and $\langle\tau\rangle$ become very large, equal, and proportional to $L$, respectively, thereby yielding Eq. (5).

Let us now make the second step. We note that the criticality properties discussed earlier are shared by all the cooperative models, for instance, the decision-making (DM) model of Ref. [5]. Let us assume that each bird is a node of a two-dimensional lattice. Let us assume that the swarm is a square of size $L$ with periodic boundary conditions. Each bird has to make a choice between flying to the right (1) or to the left $(-1)$. The decision rate is given by the parameter $g<1$. In the absence of cooperation, the probability for a given node of making a transition from the state $|1\rangle=1$, to the state $|2\rangle=-1$, or vice versa, is $g$. Each bird has four nearest neighbors. When cooperation is switched on, $\quad p_{2 \rightarrow 1}=g \exp \{K[M(1)-M(2)] / M\}$ and $p_{1 \rightarrow 2}=g \exp \{K[M(2)-M(1)] / M\}$, where $M=4$ and $M(1)$ and $M(2)$ are the numbers of nearest neighbors in the state $|1\rangle$ and $|2\rangle$, respectively. Each node generates a signal $s(t)$, with $s(t)= \pm 1$. In the absence of cooperation, the time duration of either a positive or negative signal is $1 / g$. When $K>0$ the mean time duration of a state becomes larger, and at criticality it starts increasing exponentially [5].

This model makes it easy to study the effects of the action of the danger- or resource-perceiving units. We

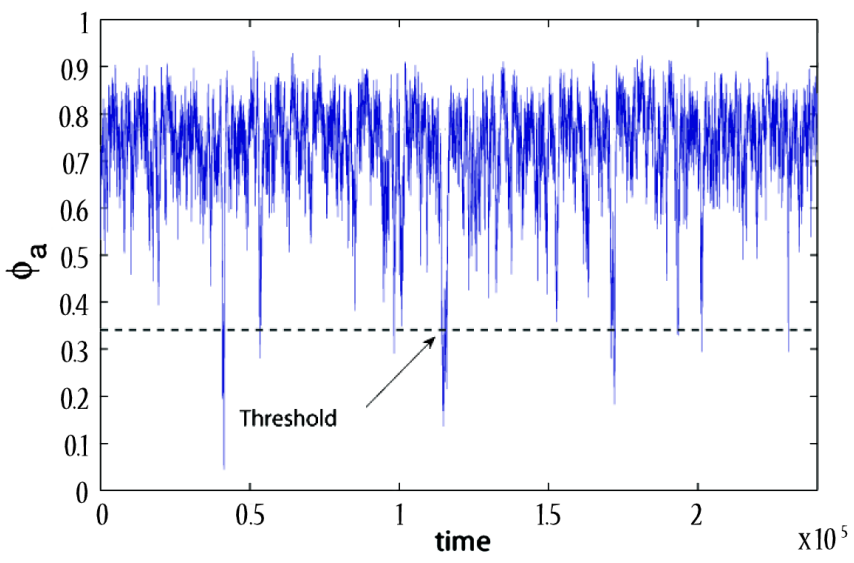

FIG. 2 (color online). Intensity of the global speed as a function of time for $N=1600$ birds for the value of noise $\eta_{c}=3.5$ corresponding to the onset of phase transition in the twodimensional case. 


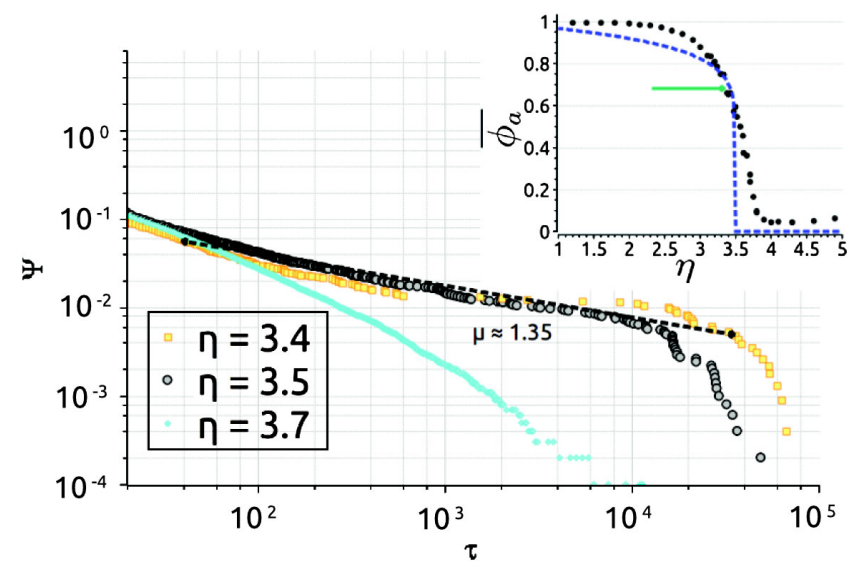

FIG. 3 (color online). Survival probability for the onedimensional bird model, at different values of the control parameter $1 / \eta$. The inset shows the time mean value of the global field $\phi_{a}(\eta)$, and the dashed line has been calculated by fitting the data with a function of the type $\phi=\left[\left(\eta_{c}-\eta\right) / \eta_{c}\right]^{\beta}$.

assume that the units of the small squares of size $\Delta L$, in the left bottom of the swarm, namely, $\Delta L^{2}$ units, are the lookout birds. From time $T$ on, we assign to them a regular and simple form of motion, representing their response to the environment, and we study the transmission of this motion to the whole swarm via the nearest-neighbor interaction. The simple form of motion that we assign to the lookout birds is based on dividing the time axis into intervals of size $\Delta t$ where they fly either to the right or to the left, and, for simplicity, we choose an alternate prescription. The top left panel of Fig. 4 shows that when the effort parameter $K$ is smaller than the critical value, the swarm is unable to follow the direction of the lookout birds. For even smaller values of the cooperation effort, the swarm does not even depart from its unperturbed behavior. The top right panel of Fig. 4 shows that when the cooperative effort is too intense, the swarm is not flexible and does not succeed in synchronizing its behavior with that of the lookout birds either. At criticality, instead (see the bottom panel of Fig. 4), the whole swarm synchronizes very well with the lookout birds.

To study this phenomenon from the information transport point of view, we imagine that the motion of the lookout birds is determined by a transmitter operator $O_{T}$ and that the flying direction, either 1 or -1 , assigned to the lookout birds for each time interval $\Delta t$, is 1 bit of information. Operator $O_{T}$ transmits the sequence $\{t(n)\}$, with $n=1,2, \ldots, B-1, B$, where $t(n)= \pm 1$ and $B$ is the total number of bits to transmit. The receiver operator $O_{R}$ observes the signal produced by a node located at a very large distance. The signal produced by the unit observed by $O_{R}$ is erratic and not much different from the random behavior of the units in isolation. However, as erratic as the single units are, at criticality the fluctuations observed by $O_{R}$ contain information on the global variable. The operator $O_{R}$ has to record this signal and make a time average. This
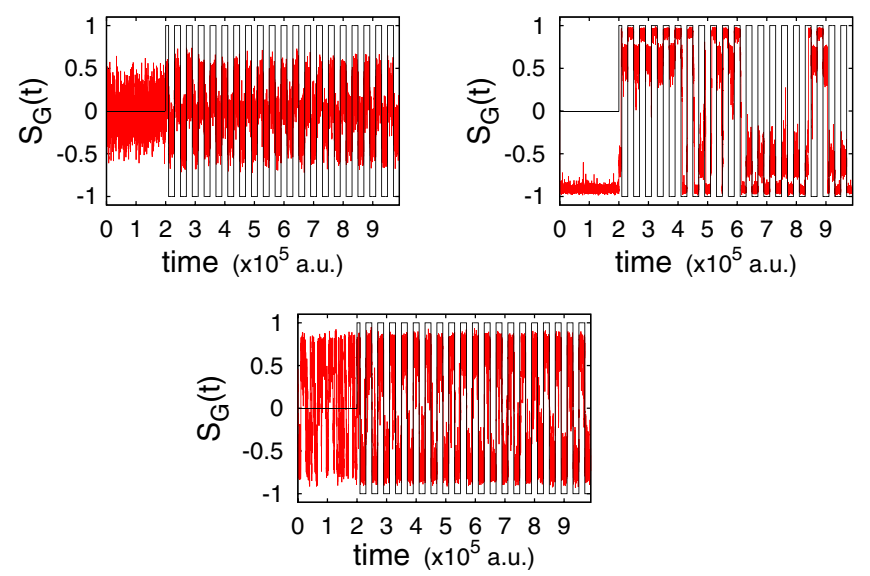

FIG. 4 (color online). Mean field of the DM model with 400 units corresponding to the nodes of a 20 by 20 regular twodimensional lattice with periodic boundary conditions. From time $t=0$ to $t=300000$, the global field is determined only by the internal cooperation among the units. At time $t=T=$ 300000 , a small cluster of 25 units moves from the state "yes," corresponding to the value of 1 , to the state "no," corresponding to the value of -1 , and so on. The black lines depict the motion of the lookout birds, with the vertical lines marking the forced abrupt transition from one to the other state. The top left, top right, and bottom panel refer to a subcritical $K=1.4$, supercritical $K=1.9$, and critical $K=1.6$ condition, respectively.

time average has to be done according to a preliminary agreement between the operator $O_{T}$ and the operator $O_{R}$. Thus, operator $O_{R}$ generates the sequence $\{b(n)\}$, where $b(n)$ is the time average of the signal $s(t)$ generated by the node under observation from time $t_{n-1}=(n-1) \Delta t$ to time $t_{n}=n \Delta t$. The transmission of the message begins at time $t_{1}=T$ and ends at time $t_{B}=B \Delta t$.

We see that at criticality (the bottom panel of Fig. 5) the sign of the averaged signal corresponds exactly to the sign of the transmitted message: a macroscopic signal reproducing perfectly the message of $O_{T}$. This perfect transmission condition is lost in both the subcritical and supercritical conditions. In the case of Fig. 5, the Euclidean distance between $T$ and $R$ is about 9 , while the distance between two units in direct contact is 1 .

To prove that the flock's response to the directions of the lookout birds is determined by the criticality-induced nonlocality, we evaluate the global correlation of the swarm according to the following procedure. For each bird of the swarm we turn the sequence $b(n)$ of Fig. 5 into the binary sequence defined by

$$
r(n)=\operatorname{sgn}[b(n)] .
$$

Then we determine the correlation with the message transmitted by $O_{T}$ :

$$
c=\frac{\sum_{n=0}^{n=B} r(n) t(n)}{B}
$$



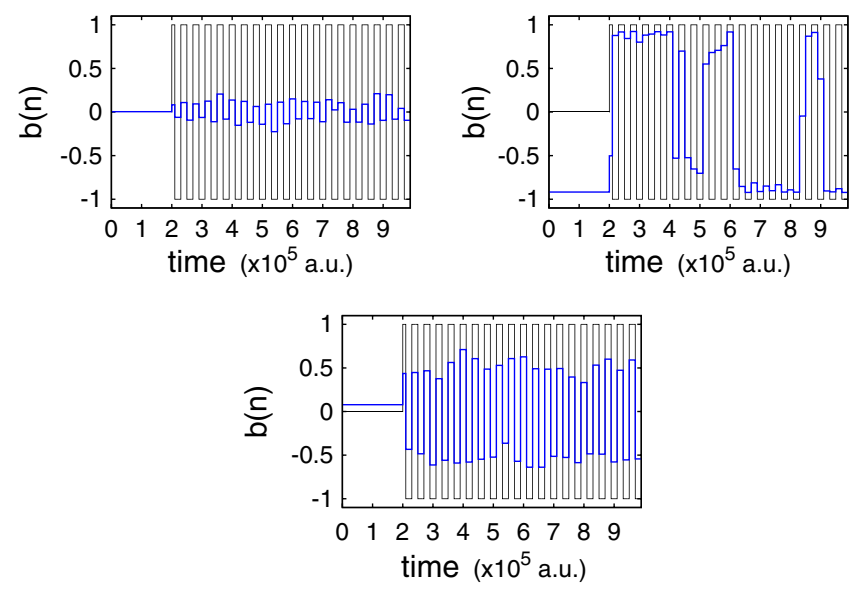

FIG. 5 (color online). This figure refers to the same condition as that of Fig. 4. Here we plot the time averages that the operator of $R$ has to make to perceive the signal.

where $B$ is the total number of transmitted bits. We evaluate this correlation for each node and determine its mean value $C$, which is plotted in Fig. 6 . We see that increasing $\Delta t$ has the effect of improving the accuracy of information transmission, which reaches its maximum at criticality, with $K_{c} \approx 1.61$. When $\Delta t=20000$, the maximum of $C$ at criticality is $C_{\max } \approx 1$, a fact implying that correlation has the maximum value for each node, regardless of its distance from the lookout birds.

There is no direct connection between $O_{T}$ and $O_{R}$. The transmission of a message from $O_{T}$ to $O_{R}$ is a consequence of the criticality-induced long-range correlation, and the time required to transmit information from $O_{T}$ to $O_{R}$ is determined by the distance between two consecutive organizational collapses.

To confirm that the DM model fits Eq. (5), we study two cases, 400 and 900 nodes. According to Eq. (5), we expect that $\langle\tau\rangle_{900}=1.5\langle\tau\rangle_{400}$. The adoption of the procedure generating the inset of Fig. 3, we find that $K_{900}=1.62$ and $K_{400}=1.61$. The corresponding $\langle\tau\rangle$ values are $\langle\tau\rangle_{900} \approx 430$ and $\langle\tau\rangle_{400} \approx 300$, in qualitative agreement with Eq. (5). This indicates that $\langle\tau\rangle$ at criticality is only 1 order of magnitude greater than its value in the absence of cooperation, $1 / g=10$, in the numerical calculations of Figs. 4-6. At criticality, $\langle\tau\rangle$ begins increasing exponentially, and for values of the control parameter $K$ moderately larger than $K_{c}$ it can become of the order of $\Delta t$, or larger. In that condition the correlation between the lookout birds and the swarm birds drops quickly to zero.

This Letter shows that the DM model shares the same properties as the bird model and sheds light into the criticality-induced information transport, thereby suggesting a communication algorithm that, as shown by the results of Fig. 5, may have important engineering

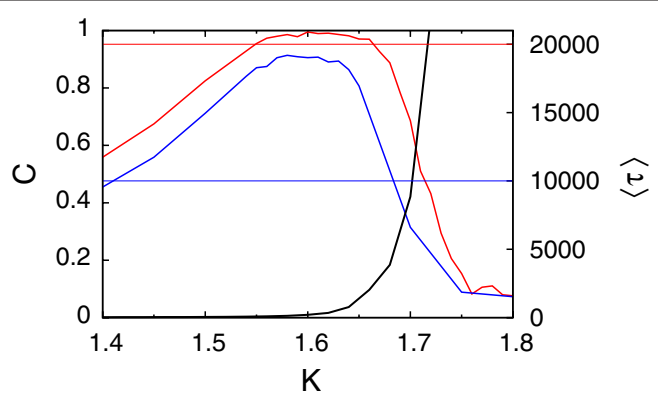

FIG. 6 (color online). Central role of criticality-induced correlation. The two bell-shaped curves denote the correlation $C$ and must be referred to the left ordinate axis. The higher corresponds to $\Delta t=20000$ and the lower to $\Delta t=10000$. The monotonically increasing curve represents $\langle\tau\rangle$ and must be referred to the right ordinate axis. The two horizontal lines are guidelines indicating the time level $\Delta t$. A 30 by 30 lattice was used to obtain these results.

applications. This efficient information transmission is closely connected to the intelligence emergence as a criticality phenomenon [11]. It is expected that working along these lines will settle the intriguing and long-standing issue of whether the communication problem rests on traveling waves or on the simultaneous action of the flock members $[1,12]$. Although the time delay suggests the questionable [12] action of information traveling waves, criticalityinduced nonlocality is the key ingredient of this process of information transmission. Increasing the swarm size has the effect of increasing the delay time, thereby making the swarm less efficient.

F. V. and P. G. thankfully acknowledge financial support from ARO and Welch through Grants No. W911NF-05-10205 and No. B-1577, respectively.

[1] I. L. Bajec and F. H. Heppner, Animal Behaviour 78, 777 (2009).

[2] A. Cavagna et al., Proc. Natl. Acad. Sci. U.S.A. 107, 11865 (2010).

[3] J. Buhl et al., Science 312, 1402 (2006).

[4] I. Couzin, Nature (London) 445, 715 (2007).

[5] M. Turalska et al., Phys. Rev. E 80, 021110 (2009); M. Turalska, B. J. West, and P. Grigolini, Phys. Rev. E 83, 061142 (2011).

[6] H. Scher and E.W. Montroll, Phys. Rev. B 12, 2455 (1975).

[7] A. Rebenshtok, E. Barkai, J. Stat. Phys. 133, 565 (2008).

[8] G. Aquino et al., Phys. Rev. Lett. 105, 040601 (2010).

[9] G. Aquino et al., Phys. Rev. E 83, 051130 (2011).

[10] T. Vicsek et al., Phys. Rev. Lett. 75, 1226 (1995).

[11] D. R. Chialvo, Nature Phys. 6, 744 (2010).

[12] W. K. Potts, Nature (London) 309, 344 (1984). 